\title{
Opioid Prescribing Habits in a Family Medicine Residency Program for the Management of Non-Cancer Pain
}

\author{
Anne Marie Thibodeaux, PharmD/MPH Candidate 2018'; Keith C. Foster, PharmD/MPH Candidate 2018'; Jessica W. Skelley, PharmD \\ $B C A C P^{2}$; Marion Sims, MD, FAAFP \\ ${ }^{1}$ Samford University McWhorter School of Pharmacy \\ ${ }^{2}$ Samford University McWhorter School of Pharmacy; Adjunct Faculty, St. Vincent's East Family Medicine Residency Program \\ ${ }^{3}$ Program Director, St. Vincent's East Family Medicine Residency Program
}

\begin{abstract}
Objectives: 1. List components of the 2016 CDC Guideline for Prescribing Opioids for Chronic Pain, 2. Describe the prescribing habits of medical residents and attending physicians within a family medicine residency program, 3. Discuss the direction of future research

Methods: A report was generated for all patients with opioids listed as a medication at Christ Health Center family medicine clinic from July 2016 to June 2017. A total of 153 patients were identified with prescriptions written for chronic non-cancer pain indications. Clinical management via a retrospective chart review was completed utilizing a standardized data collection form centered around four of twelve recommendations within the 2016 CDC Guideline for Prescribing Opioids for Chronic Pain: (1) Avoid concurrent opioid and benzodiazepine prescribing; (2) evaluate benefits and harms with patients within 1 to 4 weeks of starting opioid therapy; (3) perform urine drug testing before starting opioids and consider at least annually; and (4) offer/prescribe medication for opioid use disorder for all patients taking chronic opioids.

Results: A total of 153 prescriptions were written for chronic indications. The most common indications were chronic back pain (32.0\%), unspecified chronic pain (31.4\%), and osteoarthritis (9.8\%). Average duration of therapy was 26.6 months. Forty-two (27.5\%) patients were concurrently receiving benzodiazepine therapy. Eighteen (11.8\%) patients performed a drug test before or during therapy. Twenty-two (14.4\%) patients had documented discussion with their prescriber evaluating the benefits and harms of their opioid regimens. No patients were prescribed medication for opioid overdose.

Conclusion: Prescribing habits did not align with the four-guideline recommendations evaluated. The need for provider-focused education on current pain management practice guidelines was identified.
\end{abstract}

Keywords: opioid prescribing, primary care, non-cancer pain

\section{Introduction}

Managing non-cancer pain has become an increasingly large challenge for healthcare providers. In 2012, prescribers wrote 82.5 opioid prescriptions per 100 persons in the United States. ${ }^{1}$ As the number of opioid prescriptions has increased, so have the number of opioid related overdoses, resulting in opioidrelated deaths being five times higher today than they were 15 years ago. 1,2,3 From the state perspective, in 2012 Alabama wrote more opioid prescriptions than any other state in the country: 121 opioid prescriptions for every 100 people. ${ }^{4}$

Despite early efforts by opioid manufacturers to convince prescribers that opioids were safe to use in the majority of patient populations, it is now understood that opioid dependence has a high prevalence, with roughly $25 \%$ of patients misusing their prescribed opioids and $10 \%$ of patients developing an opioid use disorder. ${ }^{2,5}$ Costs associated with emergent treatment of opioid overdose have been on par with treatment costs of other major chronic disease states such as asthma and HIV. ${ }^{6}$

Corresponding author: Anne Marie Thibodeaux, PharmD Samford University McWhorter School of Pharmacy Email: athib32@gmail.com
Improper prescribing is a main culprit surrounding the opioid epidemic. ${ }^{1}$ In a 2016 letter from U.S. Surgeon General Dr. Vivek Murthy, more than 2 million prescribers were called upon to address the issues surrounding opioid prescribing in their practices. $^{7}$ Issues in prescribing include overlapping pain relievers, use of opioids concurrently with benzodiazepines, use of long-acting pain relievers in patients with acute pain, and prescribing higher doses than necessary. ${ }^{1}$ Volatility of pain varying from patient to patient makes managing pain control difficult, with patients with high volatility often having poor outcomes in pain management. ${ }^{8}$

As healthcare providers wrestle with providing adequate pain control while avoiding adverse effects, there has been more attention on this epidemic. As new guidelines and recommendations are published, it is important for prescribers to stay up to date and remain properly trained. One previous study compared opioid prescribing habits based on the number of CME hours obtained related to opioids. Prescribers with more CME hours related to opioid prescribing adhered to the guidelines more than those prescribers with less CME hours, illustrating the need for proper prescriber training and periodic updates as new guidelines and recommendations are published. $^{9}$ In 2016, the Centers for Disease Control and Prevention (CDC) published a guideline for prescribing opioids for chronic pain (Table 1). ${ }^{10}$ This study aims to identify current 
opioid prescribing habits for non-cancer pain in a family medicine residency program, and to evaluate if those habits align with specific recommendations within the 2016 CDC guideline.

\section{Methods}

This project was approved by the Institutional Review Board at Samford University in Birmingham, Alabama. Patients who were prescribed opioids at Christ Health Center, a family medicine residency program, were identified as the study population. A report was generated for all patients with documentation of an opioid as an active or inactive medication on the medication list between July 1, 2016 and June 30, 2017. Patients were included in the analysis if they were 18 years or older and were prescribed an opioid for a chronic indication. Patients were excluded if they were receiving their opioid prescription from an outside provider or were prescribed opioids for cancer-related pain. A chart review was conducted analyzing the following recommendations from the 2016 CDC Guideline for Prescribing Opioids for Chronic Pain: (1) avoid opioids in patients concurrently receiving benzodiazepine therapy; (2) perform a urine drug test on patients before opioid therapy and at least annually; (3) evaluate patients for benefits and harms of opioid therapy; and (4) consider a prescription for an opioid overdose medication. Results were completed using a standardized data collected form. Data were collected regarding the indication of the opioid, the duration of therapy, concomitant use of benzodiazepines, documentation of drug screening at any point in therapy, documentation of risks versus benefits for continuation of therapy, and need for an opioid overdose medication. No patients included in the study received more than one course of therapy. Because medical residents work under direct supervision of an attending physician when prescribing opioids, data were not divided between the two cohorts. Therefore, the term "prescriber" refers to both medical residents and attending physicians.

\section{Results}

After running a data report, 623 patient charts were identified as having documentation of an opioid on their medication list between July 1, 2016 and June 30, 2017. Of those identified, 153 received their prescription from a provider within the clinic for a chronic non-cancer pain indication. The average age was 61.3 years, with $71 \%$ of patients being female. The most common indication for opioid use was chronic back pain (32.0\%), followed by unspecified chronic pain (31.4\%) and osteoarthritis (9.8\%). A full list of indications can be found in Figure 1. Average duration of opioid therapy was 26.6 months.

Various aspects of care were compared to the CDC guideline for chronic pain. Forty-two (27.5\%) patients were concurrently receiving benzodiazepine therapy. Eighteen (11.8\%) patients performed a urine drug test during therapy to assess for prescribed medications and illicit substance use. Twenty-two (14.4\%) patients had documented discussion with their prescriber evaluating the benefits and harms of their opioid regimens. No patients were prescribed medication for opioid overdose.

\section{Discussion}

This retrospective chart review examined opioid prescribing habits in a family medicine residency program. Overall, prescribers in the clinic did not closely follow the recommendations provided by the $C D C$ guideline. Almost half of patients concurrently on a benzodiazepine were prescribed an opioid, only $12 \%$ received a urine drug test before or during opioid treatment, $14 \%$ were appropriately evaluated for benefits \& harms, and zero patients received a prescription for opioid overdose medication. Prescribers had been previously educated on the 2016 CDC guidelines, but our findings indicate that more education on the guidelines is warranted. In the clinic specifically, several patients have been receiving chronic opioid therapy for years, challenging prescribers to have difficult conversations with patients about their potential adjustments to treatment plans. Addressing those differences in expectations is a critical obstacle all prescribers endure, and educating them on how to have those discussions could be a vital resource to achieve the ultimate goal of creating a space where providers and patients are able to speak freely and develop a plan that best promotes patient wellness. ${ }^{11}$ Providers must also seek to develop better understanding of their own perceptions of pain management, as various biases exist that can create disparities in providing appropriate care for patients with chronic pain. ${ }^{12}$

One limitation of this study is that only the 2016 CDC Guideline for Prescribing Opioids for Chronic Pain was used to evaluate practices. The American Academy of Pain Medicine and the American Society of Anesthesiologists also provide guidelines into pain management, but they were not utilized. ${ }^{12,13}$ While these guidelines share many commonalities, certain differences exist, such as the use of benzodiazepines. Having evaluated only four of twelve specific recommendations within the CDC guideline, results could be strengthened with a future study evaluating the remaining eight recommendations. Very few electronic health records had documented evidence of discussing benefits and harms of opioid therapy with patients during the visit. However, prescribers could have had these conversations with patients but did not document the conversation, as research has indicated that underreporting and miscommunication of what should be documented in the EHR are major sources of discrepancy. ${ }^{14}$ Lastly, our small sample size of 153 and prescribers within the setting of one residency program limit its ability to be extrapolated to the larger population.

This chart review demonstrated that areas of improvement for medical residents and attending physicians exist in regard to evaluating benefits and risks of continuing opioid therapy, as well as providing treatment for possible overdose. Education opportunities need to be provided to not only understand 
guideline recommendations, but also the importance of discussing pain management expectations and treatment plans with patients.

\section{Conclusion}

Of the specific recommendations evaluated, prescribing habits did not align with the 2016 CDC Guideline for Prescribing Opioids for Chronic Pain. The need for provider-focused education on guidelines as well as management of patient expectations was identified. Prescribers would benefit from focused training in concurrent use of opioids and benzodiazepines, the appropriateness of prescribing a medication for opioid overdose, and the utility of discussing expectations with patients while evaluating benefits and harms of their treatment regimens.

Acknowledgement: None

Funding: None

Conflicts of Interest: None

Treatment of Human Subjects: Samford University and St. Vincent's Health System IRB approved this research.

Link to Streaming Media: None

\section{References}

1. Understanding the Epidemic. Centers for Disease Control and Prevention.

https://www.cdc.gov/drugoverdose/epidemic/index. html. Published December 16, 2016. Accessed April 23, 2017.

2. Dowell D, Farley T, Kunins H. Opioid analgesics - risky drugs, not risky patients. JAMA. 2013; 21:2219.

3. Kattan J, Tuazon E, Kunins $\mathrm{H}$, et al. Public Health Detailing--A Successful Strategy to Promote Judicious Opioid Analgesic Prescribing. Am J Public Health. 2016; 106:1430-1438.

4. Vital Signs: Variation Among States in Prescribing of Opioid Pain Relievers and Benzodiazepine- United States, 2012. Centers for Disease Control and Prevention. https://www.cdc.gov/mmwr/preview/mmwrhtml/m m6326a2.htm?s_cid=mm6326a2_w. Published July 4, 2014. Accessed April 23, 2017.

5. Vowles KE, McEntee ML, Julnes PS, Frohe T, Ney JP, van der Goes DN. Rates of opioid misuse, abuse, and addiction in chronic pain: a systematic review and data synthesis. Pain. 2015;156(4):569-576. doi:10.1097/01.j.pain.0000460357.01998.f1

6. Volkow ND, Frieden TR, Hyde PS, Cha SS. Medicationassisted therapies- tackling the opioid overdose epidemic. N Engl J Med. 2014; 370:2063-2066.

7. Murthy V. Ending the opioid epidemic - a call to action. N Engl J Med. 2016;(25):2413.
8. Worley MJ, Heinzerling KG, Shoptaw S, Ling W. Pain volatility and prescription opioid addiction treatment outcomes in patients with chronic pain. Exp Clin Psychopharmacol. 2015; 23:428-435.

9. McCalmont JC, Jones KD, Bennett RM, et al. Does familiarity with CDC guidelines, continuing education, and provider characteristics influence adherence to chronic pain management practices and opioid prescribing? J Opioid Manag. 2018 MarApr;14(2):103-116. doi: 10.5055/jom.2018.0437.

10. Dowell D, Haegerish TM, Chou R. CDC Guideline for Prescribing Opioids for Chronic Pain- United States, 2016. MMWR Recomm Rep. 2016; 65:1-49.

11. Lorenzetti RC, Jacques $\mathrm{CH}$, Donovan C, Cottrell S, Buck J. Managing difficult encounters: understanding physician, patient, and situational factors. Am Fam Physician. 2013; 87:419-425.

12. American Academy of Pain Medicine. Use of opioids for the treatment of chronic pain. Pain Med. 2013; 14.

13. Rosenquist RW, Benzon HT, Connis RT, et al. Practice guidelines for chronic pain management. Anesthesiology. 2010; 112:1-24.

14. Vowles KE, McEntee ML, Julnes PS, Frohe T, Ney JP, van der Goes DN. Rates of opioid misuse, abuse, and addiction in chronic pain: a systematic review and data synthesis. Pain. 2015;156(4):569-576. doi:10.1097/01.j.pain.0000460357.01998.f1 


\section{Appendix}

Table 1. Recommendations from the 2016 CDC Guideline for Prescribing Opioids for Chronic Pain

\section{Determining When to Initiate or Continue Opioids for Chronic Pain}

Nonpharmacologic therapy and nonopioid pharmacologic therapy are preferred for chronic pain. Clinicians should consider opioid therapy only if expected benefits for both pain and function are anticipated to outweigh risks to the patient. If opioids are used, they should be combined with nonpharmacologic therapy and nonopioid pharmacologic therapy, as appropriate.

(Recommendation category: A, Evidence type: 3 )

Before starting opioid therapy, clinicians should establish treatment goals with all patients, including realistic goals for pain and function, and should consider how opioid therapy will be discontinued if benefits do not outweigh risks. Clinicians should continue opioid therapy only if there is clinically meaningful improvement in pain and function that outweighs risks to patient safety. (Recommendation category: A, Evidence type: 4)

Before starting and periodically during opioid therapy, clinicians should discuss with patients known risks and realistic benefits of opioid therapy and patient and clinician responsibilities for managing therapy. (Recommendation category: A, Evidence type: 3 )

\section{Opioid Selection, Dosage, Duration, Follow-Up, and Discontinuation}

When starting opioid therapy for chronic pain, clinicians should prescribe immediate-release opioids instead of extendedrelease/long-acting (ER/LA) opioids. (Recommendation category: A, Evidence type: 4)

When opioids are started, clinicians should prescribe the lowest effective dosage. Clinicians should use caution when prescribing opioids at any dosage, should carefully reassess evidence of individual benefits and risks when considering increasing dosage to $\geq 50$ morphine milligram equivalents (MME)/day, and should avoid increasing dosage to $\geq 90 \mathrm{MME} /$ day or carefully justify a decision to titrate dosage to $\geq 90 \mathrm{MME} /$ day. (Recommendation category: A, Evidence type: 3 )

Long-term opioid use often begins with treatment of acute pain. When opioids are used for acute pain, clinicians should prescribe the lowest effective dose of immediate-release opioids and should prescribe no greater quantity than needed for the expected duration of pain severe enough to require opioids. Three days or less will often be sufficient; more than seven days will rarely be needed. (Recommendation category: A, Evidence type: 4)

Clinicians should evaluate benefits and harms with patients within 1 to 4 weeks of starting opioid therapy for chronic pain or of dose escalation. Clinicians should evaluate benefits and harms of continued therapy with patients every 3 months or more frequently. If benefits do not outweigh harms of continued opioid therapy, clinicians should optimize other therapies and work with patients to taper opioids to lower dosages or to taper and discontinue opioids. * (Recommendation category: A, Evidence type: 4)

\section{Assessing Risk and Addressing Harms}

Clinicians should review the patient's history of controlled substance prescriptions using state prescription drug monitoring program (PDMP) data to determine whether the patient is receiving opioid dosages or dangerous combinations that put him or her at high risk for overdose. Clinicians should review PDMP data when starting opioid therapy for chronic pain and periodically during opioid therapy for chronic pain, ranging from every prescription to every 3 months. (Recommendation category: A, Evidence type: 4)

When prescribing opioids for chronic pain, clinicians should use urine drug testing before starting opioid therapy and consider urine drug testing at least annually to assess for prescribed medications as well as other controlled prescription drugs and illicit drugs. * (Recommendation category: B, Evidence type: 4)

Clinicians should avoid prescribing opioid pain medication and benzodiazepines concurrently whenever possible.* (Recommendation category: A, Evidence type: 3)

Clinicians should offer or arrange evidence-based treatment (usually medication-assisted treatment with buprenorphine or methadone in combination with behavioral therapies) for patients with opioid use disorder.* (Recommendation category: A, Evidence type: 2)

\footnotetext{
${ }^{*}$ Recommendation evaluated in this study
} 
Figure

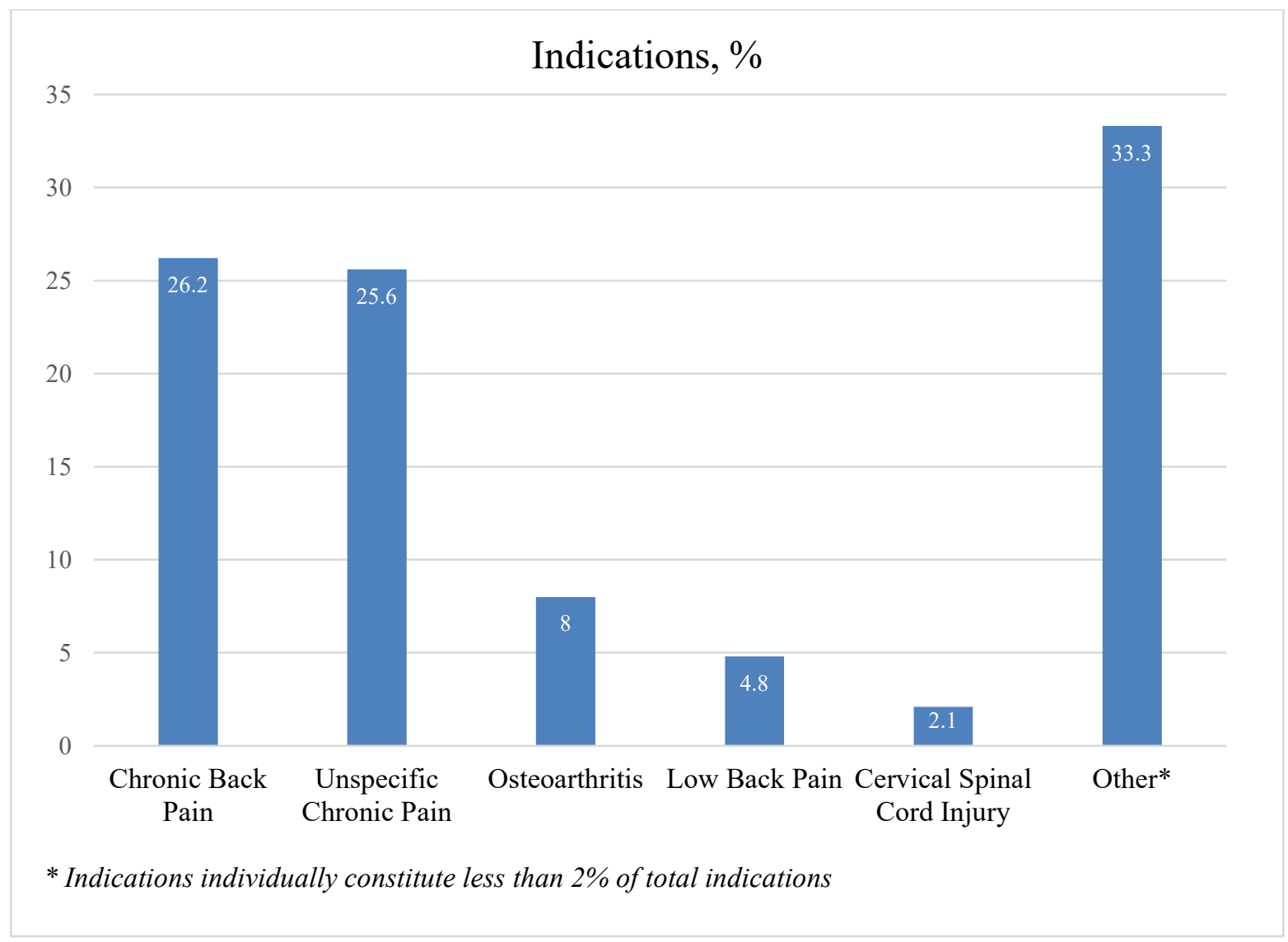

